\title{
Les cellules péritubulaires
}

Dans le testicule des mammifères une lamina propria sépare les tubules séminifères du tissu interstitiel. Cette lamina propria contient des éléments cellulaires ainsi que des éléments acellulaires [1, 2]. Immédiatement en dessous de l'épithélium séminifère on retrouve une membrane basale, suivie d'une zone plus importante de matériel extracellulaire riche en collagène. Enfin, les cellules caractéristiques de la lamina propria, notamment les cellules péritubulaires myoïdes, se situent à la périphérie. Des techniques récentes permettant d'étudier ces cellules in vitro ont souligné leur rôle important dans la différenciation tubulaire et dans le maintien de la structure et de la fonction des cellules de Sertoli.

\section{Aspects morphologiques et développement}

Chez le rat et la souris, les cellules péritubulaires forment une couche continue de cellules polygonales avec des caractéristiques myo-épithéliales. Chez les mammifères plus évolués comme l'homme, plusieurs couches concentriques (2 à 5 ) de cellules myoïdes étoilées et extrêmement plates peuvent être distinguées. Chaque couche est séparée de la suivante par une zone de matériel extracellulaire contenant du collagène, des fibres d'élastine et de microfibrilles caractéristiques (figure 1).

Chez toutes les espèces, la morphologie des cellules péritubulaires est très semblable $[1,3,4]$. Cette morphologie est intermédiaire entre celle d'un fibroblaste et celle d'une cellule myoïde. Particulièrement importants sont les faisceaux de fins filaments occupant la périphérie du cytoplasme. Ils sont supposés jouer un rôle dans la contractilité des cellules myoïdes et semblent s'accrocher aux densifications cytoplasmiques typiques. En outre, le cytoplasme con- tient des micro-vésicules pinocytotiques et un réticulum endoplasmique rugueux assez abondant. Dans le testicule humain, une membrane basale discontinue entoure les cellules myoïdes. Chez le rat, des fragments de membrane basale apparaissent spécifiquement au bord interstitiel des cellules péritubulaires, ce qui suggère une certaine polarisation. A l'extérieur des cellules péritubulaires on peut retrouver des fibroblastes, peut-être les précurseurs des cellules myoïdes ou des cellules endothéliales du système lymphatique testiculaire.

L'origine mésenchymateuse des cellules péritubulaires a bien été établie. En effet, très tôt dans le développement des gonades et parallèlement à la différenciation épithéliale des cellules de Sertoli, on note des changements au niveau des cellules mésenchymateuses progénitrices qui se transforment progressivement en cellules péritubulaires [3-5]. Néanmoins, ces jeunes cellules péritubulaires ressemblent plutôt à des fibroblastes contenant une abondance de ribosomes libres et de réticulum endoplasmique rugueux. Les citernes du réticulum endoplasmique sont parfois dilatées et l'appareil de Golgi est bien développé. Pendant les premières semaines de développement postnatal du rat, les cellules les plus proches des tubules séminifères s'allongent de plus en plus. Dès le huitième jour, des faisceaux de filaments typiques font leur apparition dans le cytoplasme et des fragments de membrane basale sont déposés à la périphérie de la couche de cellules péritubulaires. Un peu plus tard, on observe l'apparition d'une réaction positive pour la phosphatase alkaline et, parallèlement, celle de contractions spontanées dans des tubules isolés. A l'âge de 22 jours, les cellules péritubulaires ont acquis leur morphologie adulte.
La différenciation des cellules myoïdes est sans doute sous contrôle hypophysaire [4]. La transplantation de tubules séminifères provenant de rats immatures dans des testicules de rats adultes démontre, en effet, une différenciation progressive des cellules myoïdes. L'hypophysectomie des animaux récepteurs empêche cette différenciation. Comme les cellules myoïdes ne possèdent pas de récepteurs, ni pour la $\mathrm{LH}$, ni pour la $\mathrm{FSH}$, les effets des hormones hypophysaires sont probablement indirects. En effet, l'administration d'androgènes annule en partie l'effet de l'hypophysectomie. Néanmoins, outre les androgènes, probablement d'autres signaux provenant de l'épithélium séminifère contribuent à la maturation des cellules péritubulaires [4].

\section{Aspects fonctionnels}

L'universalité des cellules péritubulaires myoïdes ainsi que le contrôle précis de leur différenciation suggèrent qu'elles représentent un élément fonctionnel essentiel dans le testicule des mammifères. Leur rôle exact reste cependant mal compris (Tableau I).

Chez certaines espèces telles que le rat, les cellules péritubulaires représentent un élément constitutif de la barrière hémato-testiculaire [6]. Elles possèdent des jonctions serrées et limitent la diffusion de traceurs calibrés marqués au carbone et au thorium et, jusqu'à un certain point, la ferritine $(9 \mathrm{~nm})$ et le lanthanum vers le compartiment tubulaire. Chez les primates, une telle fonction est improbable. En tout cas, les jonctions serrées intersertoliennes restent le composant principal de la barrière hémato-testiculaire. En outre, les contractions des tubules séminifères sont, sans doute, sous la responsabilité des cellules péritubulaires. De nouveau, ce phénomène a été étudié 
surtout chez le rat, le lapin et le chien. Les contractions sont, au moins en partie, sous le contrôle conjoint de l'ocytocine et des androgènes [7]. L'action des androgènes est probablement directe. En effet, le récepteur des androgènes est assez abondant dans les cellules myoïdes [8]. En revanche, le mode d'action de l'ocytocine reste mal compris car, jusqu'à maintenant, personne n'a pu démontrer la présence du récepteur de l'ocytocine dans les cellules péritubulaires. Celles-ci jouent sans doute un rôle important dans la production, le dépôt et la restructuration de la matière extracellulaire entourant les tubules séminifères. L'efficacité de tous ces processus exige une coopération étroite entre les cellules péritubulaires et les cellules de Sertoli $[2,9,10]$. Il est important de noter que la pathologie testiculaire est souvent accompagnée de changements importants dans la structure de la lamina propria [11]. Typiquement, on observe un élargissement de la zone riche en collagène située entre la membrane basale de l'épithélium séminifère et les cellules péritubulaires, ou de la zone entre les couches successives de cellules myoïdes. Dans des cas extrêmes, de la matière hyaline remplace les structures tubulaires. Enfin, les cellules péritubulaires déploient toute une gamme d'interactions paracrines avec les cellules voi- sines [12, 13]. Notre compréhension actuelle de ces interactions est surtout le résultat d'études sur des cellules isolées et cultivées in vitro.

\section{Etudes sur des cellules péritubulaires} cultivées

L'usage de cellules péritubulaires isolées et cultivées in vitro a considérablement contribué à notre connaissance actuelle de leur "sociologie cellulaire " et surtout de leurs interactions avec des cellules de Sertoli. Il faut, cependant, noter que la majorité de ces études a été effectuée à l'aide de préparations de cellules dont la nature exacte reste mal comprise. La caractéristique la plus spécifique et la plus marquée des cellules

\begin{tabular}{|c|}
\hline Tableau I \\
FONCTIONS DES CELLULES \\
PÉRITUBULAIRES \\
\hline Élément de structure \\
Contractilité des tubules \\
Barrière hémato-testiculaire \\
Dépôt et transformation \\
de la matrice extracellulaire \\
Morphogenèse des tubules \\
Différenciation et maintien \\
de la fonction des cellules \\
de Sertoli
\end{tabular}

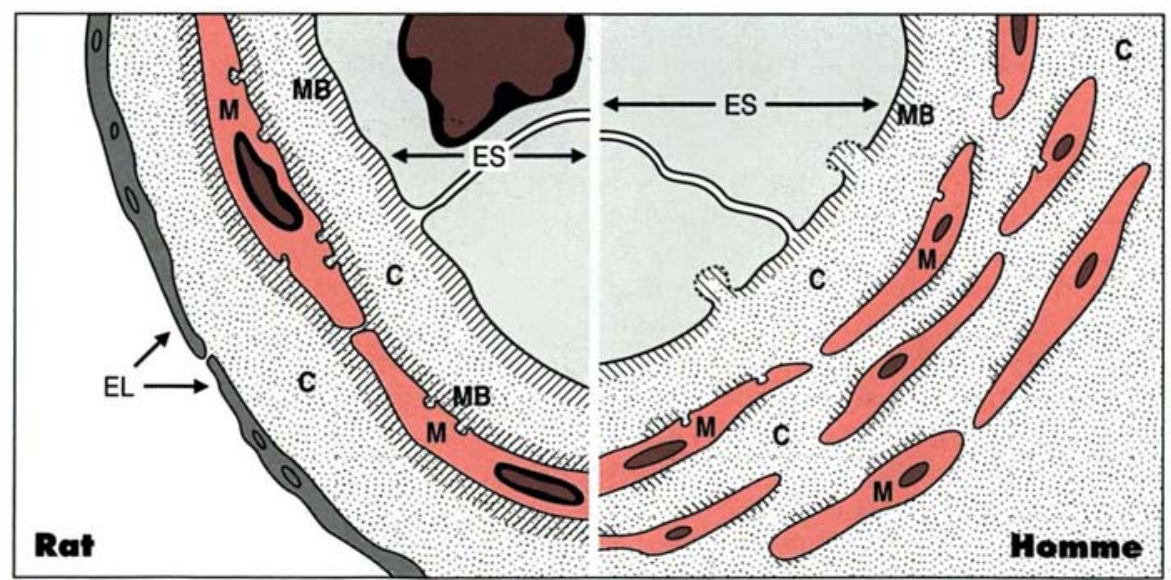

Figure 1. Structure de la lamina propria chez le rat et chez l'homme. Abréviations: $E S$, épithélium séminifère; $M B$, membrane basale; $M$, cellules péritubulaires myoïdes; $E L$, endothélium lymphatique; $C$, zone riche en collagè- péritubulaires cultivées in vitro est probablement leur réaction positive vis-à-vis d'anticorps reconnaissant l'isof orme $\alpha$ de l'actine des muscles lisses [14]. En revanche, in vitro, seule une fraction de ces cellules présente une réaction positive vis-à-vis d'autres marqueurs comme la desmine, ou la phosphatase alkaline, marqueurs qui sont clairement présents dans des cellules myoïdes in situ. Les raisons de cette discordance restent obscures. On peut imaginer que les conditions de culture et de subculture engendrent la sélection d'un type de cellules ressemblant à des myofibroblastes et présent dans le testicule intact comme une cellule mésenchymateuse progénitrice peu différenciée. Alternativement, la rupture des liens physiologiques entre les cellules péritubulaires et les cellules voisines pourrait induire un phénotype moins différencié. Quoi qu'il en soit, les cellules cultivées gardent plusieures qualités attribuées aux cellules myoïdes in vivo.

In vitro, aussi bien que in vivo, on peut observer des interactions réciproques entre les cellules mésenchymateuses péritubulaires et les cellules épithéliales de Sertoli. Ces interactions mésenchymateuses-épithéliales se traduisent par des changements morphologiques et biochimiques et jouent un rôle important dans le développement du testicule et dans le soutien de sa fonction adulte.

Morphologiquement, la mise en coculture de cellules péritubulaires et de cellules de Sertoli dans des conditions appropriées entraîne une reconstitution partielle de la différenciation foetale des cordons testiculaires [15]. Tout d'abord, on voit les cellules de Sertoli se regrouper et former des plaques et des îlots séparés par des cordons de cellules péritubulaires. Ces îlots se réunissent et produisent des rehaussements qui se transformeront finalement en boules et en structures tubulaires dépourvues, bien sûr, de cellules germinales. Il est intéressant de noter que, tout comme in vivo, la formation de ces structures tubulaires est inhibée par l'AMPc [16].

Au niveau biochimique, la coculture de cellules péritubulaires et de cellules de Sertoli prolonge la survie des 
deux populations [15]. De plus, elle augmente et prolonge la sécrétion de l'ABP (androgen binding protein) et de la transferrine $[17,18]$, deux protéines produites par les cellules de Sertoli, et modifie la sécrétion vectorielle de protéines par ces dernières [10]. Finalement, sous certaines conditions, la coculture augmente la réponse des cellules de Sertoli aux androgènes [17-19].

Au moins deux méchanismes d'interaction sont responsables de ces effets morphologiques et fonctionnels, notamment des interactions liées à l'environnement relayées par des composants de la matrice extracellulaire et des interactions paracrines relayées par des facteurs de croissance [12].

- Interactions liées à l'environnement. Les cellules péritubulaires comme les cellules de Sertoli ont la capacité de produire des éléments individuels de la matrice extracellulaire $[2,9]$. Elles synthétisent du collagène (type I et type IV), de la laminine, de la fibronectine et des protéoglycanes de haut poids moléculaire riches en chondroïtine. En revanche, les cellules de Sertoli représentent une source de collagène (type IV), de laminine et de protéoglycanes riches en chondroïtine ou en héparine. En monoculture, ces composants sont sécrétés. Ils ne forment pas une matrice organisée. Mais, lorsque les cellules péritubulaires et les cellules de Sertoli sont cocultivées, on observe le dépôt d'une matrice structurée [9, 16]. Les mécanismes gouvernant la coopération entre les cellules de Sertoli et les cellules péritubulaires restent mal compris. Il existe toutefois des indications que la production des composants individuels de la matrice extracellulaire est contrôlée, en partie, par cette matrice elle-même et, en partie, par des facteurs paracrines encore inconnus.

La matrice extracellulaire exerce des effets remarquables sur la morphologie des cellules de Sertoli cultivées in vitro. Au contraire des cellules cultivées sur des supports artificiels, elles gardent leur phénotype en "colonne" avec un noyau basal et des jonctions serrées basolatérales [10]. Ces changements morphologiques sont accompagnés d'une polarisation $\mathrm{m} / \mathrm{s} n^{\circ} 4$, vol. 11, avril 95 de la fonction sécrétoire et d'une augmentation de la sécrétion de la transferrine et de l'ABP, bien que l'ampleur de cet effet reste discutable. De même, des cellules péritubulaires cultivées sur une biomatrice provenant de tubules séminifères cessent de proliférer et gardent leur histotype myo-épithélial observé dans le testicule intact [20].

In vivo, la production de composants de la matière extracellulaire est surtout observée pendant la morphogenèse et le développement du testicule [2]. L'ARN messager de la fibronectine, par exemple, est abondant pendant les deux ou trois premières semaines de la vie postnatale mais devient minimal après cette période. La production de matrice extracellulaire peut être réinitiée par des lésions testiculaires ou par la mise en culture des cellules concernées. Cependant, même chez l'animal adulte, une transformation constante de la matrice extracellulaire peut être observée, liée, entre autres, au passage des spermatocytes par la barrière hémato-testiculaire. Dans ce contexte, il faut noter la production de diverses protéases et inhibiteurs de protéases par les cellules péritubulaires et par les cellules de Sertoli. Ces dernières sécrètent, par exemple, l'activateur du plasminogène de type tissulaire tandis que les cellules péritubulaires produisent l'inhibiteur de cet activateur à côté d'autres protéases comme la collagénase type $A[2,12]$.

- Interactions paracrines. Un nombre surprenant de facteurs de croissance est produit par et/ou agit sur les cellules péritubulaires $[12,13]$. Les $T a$ bleaux II et III tentent de résumer notre connaissance actuelle. Certains de ces facteurs peuvent évidemment agir de façon autocrine mais, en outre, il existe sans doute tout un réseau d'interactions paracrines avec des cellules voisines comme les cellules de Sertoli, les cellules de Leydig et d'autres cellules interstitielles. Ces interactions sont extrêmement complexes et une discussion plus détaillée peut être trouvée dans d'autres revues de ce numéro. Nous nous limiterons ici à un aspect très remarquable, le rôle potentiel des

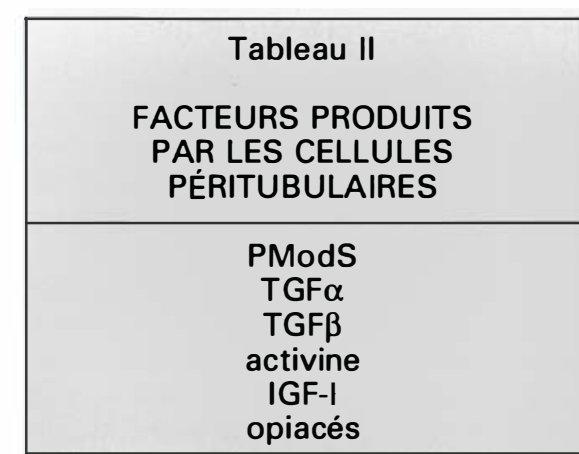

Abréviations: TGF: transforming growth factor IGF: insulin-like growth factor; PModS: peritubular factor modulating Sertoli cell function.

\begin{tabular}{|c|}
\hline Tableau III \\
FACTEURS PARACRINES \\
RÉGLANT L'ACTIVITÉ \\
DES CELLULES PÉRITUBULAIRES \\
\hline androgènes \\
TGF $\alpha$ \\
TGF $\beta$ \\
activine \\
PDGF \\
endothéline \\
interleukine 1 \\
NGF \\
ocytocine
\end{tabular}

Abréviations: PDGF: platelet derived growth factor ; TGF : transforming growth factor ; NGF: nerve growth factor.

cellules péritubulaires comme médiateurs de certains effets intratesticulaires des androgènes [12, 13, 19]. Il a été bien établi que les cellules péritubulaires ont des quantités importantes de récepteurs des androgènes et qu'elles augmentent encore, au moins in vitro, à la suite de l'exposition aux androgènes [8]. Le récepteur de l'androgène se retrouve aussi dans des cellules de Sertoli cultivées, bien que la réponse de ces dernières aux androgènes soit plutôt faible. La mise en coculture de cellules péritubulaires et de cellules de Sertoli entraîne des changements fonctionnels importańts dans les cellules de Sertoli. La production basale d'un nombre de protéines comme l'ABP, la transferrine et l'inhibine est augmentée $[8,17,18]$ et la réponse aux androgènes de ces mêmes protéines est amplifiée $[17,18]$. Cette sensibilisation aux androgènes ne requiert pas 
de contacts directs entre les cellules. En effet, des effets similaires sont observés lorsque les cellules de Sertoli sont exposées à des milieux de cultures provenant de cellules péritubulaires cultivées en absence ou en présence d'androgènes. Ces observations ont conduit à l'hypothèse que les cellules péritubulaires secrètent, sous l'influence des androgènes, un facteur qui agit comme un médiateur ou un modulateur des actions des androgènes sur les cellules de Sertoli. Le facteur concerné est généralement appelé PModS (peritubular factors that modulate Sertoli cell function) [17]. Deux protéines - PModS-A et PModS-B - ayant l'activité attendue ont été purifiées et partiellement caractérisées par Skinner et al. [21]. Ces protéines ont respectivement un poids moléculaire de $56 \mathrm{~K}$ (PModSA) et de $59 \mathrm{~K}$ (PModS-B). Un facteur avec des caractéristiques semblables mais avec un poids moléculaire un peu plus bas (40-45 K) a été décrit par notre laboratoire [18]. De plus, nous avons pu démontrer qu'une protéine avec la même activité biologique et les mêmes caractéristiques biochimiques est sécrétée par des cellules stromales de la prostate cultivées in vitro $[18,19]$. Ces observations ouvrent la possibilité que PModS pourrait correspondre à un médiateur ubiquitaire produit par des cellules mésenchymateuses et agissant sur des cellules épithéliales. Une telle hypothèse est attirante car, pendant la morphogenèse de plusieurs organes du système urogénital, le récepteur des androgènes et l'action des androgènes se retrouvent dans le tissu mésenchymateux plutôt que dans les cellules épithéliales. Il faut noter, cependant, que l'identité exacte de $\mathrm{PModS}$ reste inconnue à ce moment et, de ce fait, les relations qui pourraient exister entre les différentes formes de PModS décrites. En outre, il reste à démontrer que PModS agit bien comme un médiateur de l'action des androgènes in vivo et non seulement dans des systèmes de cellules cultivées in vitro. Finalement, il y a des indications qu'outre PModS il existe d'autres médiateurs d'actions indirectes d'androgènes comme par exemple le $k e$ -

\section{Conclusion}

En terme quantitatif, les cellules péritubulaires ne représentent qu'une fraction minimale de la population cellulaire du testicule adulte. Néanmoins, elles présentent un phénotype très spécialisé et un spectre d'action et d'interaction remarquable. Leur position stratégique à l'interface du compartiment interstitiel et du compartiment tubulaire et leurs interactions réciproques avec des cellules voisines et surtout avec des cellules de Sertoli confèrent à ces cellules myoïdes un rôle pivot dans le développement et le maintien de la fonction du compartiment tubulaire et des cellules de Sertoli, chez le mammifère adulte

\section{Guido Verhoeven \\ Eef Hoeben}

Laboratory for experimental medicine and endocrinology, Onderwijs en Navorsing, Gasthuisberg, B-3000 Leuven, Belgique.

\section{RÉFÉRENCES}

1. Bustos-Obregon E. Ultrastructure and function of the lamina propria of mammalian seminiferous tubules. Andrologia 1976 ; $8: 179-85$.

2. Dym M. Basement membrane regulation of Sertoli cells. Endocrinol Rev 1994; 15 : 102-15.

3. Leeson CR, Leeson TS. The postnatal development and differentiation of the boundary tissue of the seminiferous tubule of the rat. Anat Rec $1963 ; 147: 243-60$.

4. Bressler RS, Ross MH. Differentiation of peritubular myoid cells of the testis: effects of intratesticular implantation of newborn mouse testes into normal and hypophysectomized adults. Biol Reprod $1972 ; 6$ : 148-59.

5. Frödjman K, Paranko J, Virtanen I, Pelliniemi LJ. Intermediate filaments and epithelial differentiation of male rat embryonic gonad. Differentiation $1992 ; 50: 113-23$.

6. Ploën L, Setchell P. Blood-testis barriers revisited a homage to Lennart Nicander. Int J Androl $1992 ; 15: 1-4$.

7. Hovatta O. Effect of androgens and antiandrogens on the development of the myoid cells of the rat seminiferous tubules (organ culture). Z Zellforsch 1972; 131 : 299308.
8. Verhoeven G, Cailleau J. Testicular peritubular cells secrete a protein under androgen control that inhibits induction of aromatase activity in Sertoli cells. Endocrinology $1988 ; 123: 2100-10$.

9. Skinner MK, Tung PS, Fritz IB. Cooperativity between Sertoli cells and testicular peritubular cells in the production and deposition of extracellular matrix components. J Cell Biol 1985 ; 100 : 1941-47.

10. Dym M, Hadley MA, Djakiew D, Byers SW. Differentiation and polarized function of Sertoli cells in vitro. Adv Exp Med Biol $1987 ; 219: 515-33$

11. de Kretser DM, Kerr JB, Paulsen CA The peritubular tissue in the normal and pathological human testis. An ultrastructural study. Biol Reprod 1975; 12 : 317-24.

12. Skinner MK. Cell-cell interactions in the testis. Endocrinol Rev 1991 ; $12: 45-77$.

13. Verhoeven G. Local control systems within the testis. Bailliere's Clinical Endocrinology and Metabolism 1992 ; 6:313-33.

14. Tung PS, Fritz IB. Characterization of rat testicular peritubular myoid cells in culture: $\alpha$-smooth muscle isoactin is a specific differentiation marker. Biol Reprod 1990; 42 : 351-65.

15. Tung PS, Fritz IB. Interactions of Sertoli cells with myoid cells in vitro. Biol Reprod $1980 ; 23: 207-17$

16. Tung PS, Fritz IB. Morphogenetic restructuring and formation of basement membranes by Sertoli cells and testis peritubular cells in coculture: inhibition of the morphogenetic cascade by cyclic AMP derivatives and by blocking direct cell contact. Dev Biol 1987; 120: 139-53.

17. Skinner MK, Fritz IB. Testicular peritubular cells secrete a protein under androgen control that modulates Sertoli cell functions. Proc Natl Acad Sci USA 1985; 82 : 1148.

18. Swinnen K, Cailleau J, Heyns W, Verhoeven G. Prostatic stromal cells and testicular peritubular cells produce similar paracrine mediators of androgen action. Endocrinology $1990 ; 126: 142-50$.

19. Verhoeven G, Swinnen K, Cailleau J, Deboel L, Rombauts L, Heyns W. The role of cell-cell interactions in androgen action. $J$ Steroid Biochem Mol Biol 1992 ; 41 : $487-94$

20. Tung PS, Fritz IB. Cell-substratum and cell-cell interactions promote testicular peritubular myoid cell histotypic expression in vitro. Dev Biol 1986; 115 : 155-70.

21. Skinner MK, Fetterolf PM, Anthony CT. Purification of a paracrine factor, P-Mod-S, produced by testicular peritubular cells that modulates Sertoli cell function. J Biol Chem $1988 ; 263: 2884-90$.

\section{TIRÉS À PART}

G. Verhoeven 